\title{
THE EFFECT OF NOTCHES ON THE FATIGUE LIFE OF A NICKEL-BASE GAS TURBINE DISK MATERIAL
}

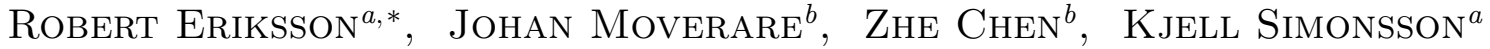 \\ ${ }^{a}$ Division of Solid Mechanics, Linköpings universitet, 58183 Linköping, Sweden \\ ${ }^{b}$ Division of Engineering Materials, Linköpings universitet, 58183 Linköping, Sweden \\ * corresponding author: robert.eriksson@liu.se
}

\begin{abstract}
Gas turbine disks carry significant load under high temperatures and may be subject to fatigue failure. Disks contain several notches in the form of the fir tree blade attachments. Low cycle fatigue tests were performed on blunt notch compact tension specimens made from alloy 718 . The results indicated that notch support needed to be incorporated not to cause an overly conservative life prediction. The notch support diminished as the plastic strain range decreased, indicating that notch support is only present in the low cycle fatigue regime. A critical distance approach was applied to account for the notch support. An equation relating the critical distance to the notch root stress was derived. The chosen life model was formulated in terms of a variation on the Smith-Watson-Topper (SWT) parameter. The modified SWT parameter taken at the critical distance was used in a life model calibrated for smooth specimens to successfully predict the fatigue life of notched specimens.
\end{abstract}

KEYwORDs: Fatigue, notch effect, gas turbines, disk alloy.

\section{INTRODUCTION}

Gas turbine disks, in the turbine section, carry significant load under intermediate to high temperatures 1]. One, of many, critical aspects from a design point of view, is to ensure the disc does not fail through fatigue. In particular, the fir tree like blade attachments account for stress concentrations which may initiate cracks in the disk.

Although notches may promote crack initiation, there is also a tendency for conventional lifing methods to underestimate the fatigue life of the component if the lifing method is applied using the notch root stress. The phenomenon is sometimes referred to as notch support [2, 3] and arises from the fact that (typically) a very small material volume is exposed to the high notch root stress; ahead of the notch, stresses typically drop significantly.

The current desire to reduce conservatism in design against fatigue (especially in the energy sector) [3] calls for notch support to be investigated and incorporated in lifing methods in an industrially applicable manner.

In the current work, a method for incorporating notch support is applied to notched specimens made from the Ni base alloy 718 subjected to low cycle fatigue (LCF). The theory of critical distances is taken as basis for the suggested lifing method.

\subsection{THEORY OF CRITICAL DISTANCES}

The theory of critical distances [4] accounts for the steep stress gradient at notches by suggesting that the failure of a notched specimen or component is more accurately based on the stress some distance from the notch root. That is, failure is assumed to occur when the stress, $\sigma(r)$, a distance $r$ from the notch root reaches a critical value, $\sigma_{0}$, such that

$$
\sigma(r)=\sigma_{0} \text { at failure }
$$

where $r$ is the critical distance.

For monotonic failure, the critical distance takes the form 4

$$
r=\frac{1}{2 \pi}\left(\frac{K_{c}}{\sigma_{0}}\right)^{2}
$$

with $K_{c}$ being the fracture toughness and $\sigma_{0}$ a strength parameter somewhat similar to the ultimate tensile strength.

The theory of critical distances has also been extended to account for fatigue failure. For high cycle fatigue $(\mathrm{HCF})$, with respect to the fatigue limit, the critical distance becomes 4

$$
r=\frac{1}{2 \pi}\left(\frac{K_{t h}}{\sigma_{0}}\right)^{2}
$$

with $K_{t h}$ being the threshold stress intensity factor for crack growth and $\sigma_{0}$ a strength parameter somewhat similar to the endurance limit.

For finite fatigue lives, it has been suggested that the critical distance should take the form [4]

$$
N_{f}=C_{1} r^{c_{1}}
$$

where $N_{f}$ is the number of cycles to failure and $C_{1}$ and $c_{1}$ are fitting parameters. Equation 4 has also been applied to low cycle fatigue of notched specimens made from a Ni-based single-crystal alloy at $500{ }^{\circ} \mathrm{C}$ [5].

Although Eqn. 4 has been demonstrated to work for LCF, it is inconvenient to use for life predictions 
as $N_{f}$ is not known a priori, requiring an iterative process for the fatigue life to be calculated.

Eriksson et al. [6] demonstrated that the critical distance could also be based on the notch root plastic strain range, $\Delta \varepsilon_{p}^{\text {root }}$, via a simple power law

$$
r=p\left(\Delta \varepsilon_{p}^{r o o t}\right)^{q}
$$

where $p$ and $q$ are fitting parameters. The rationale being that the notch root strain range correlates to the strain range further into the material.

In the current work, the method suggested by Eriksson et al. 6] will be further explored and a rationale for why the critical distance should correlate with notch root conditions will be provided. It will be shown that the critical distance can also be correlated to the notch root stress taken from a linear elastic finite element analysis (FEA).

\section{MATERIAL BEHAVIOUR}

\subsection{MATERIAL MODEL}

A simple material description, which sufficiently well captures strain ranges and mean stresses, was adopted. A two-step procedure was used to calculate needed load parameters: 1) a set of equations are used to determine the maximum stress and 2) another set of equations are used to determine the strain range. Previously published data for alloy 718 were used to describe the studied alloy, see Moverare et al. [7]. The data in Moverare et al. [7] were established in LCF tests performed in strain control with $R_{\varepsilon}=0$.

Firstly, the maximum stress is determined. For $450^{\circ} \mathrm{C}$, the maximum stress, $\sigma_{\max }$, is determined from

$$
\sigma_{\max }= \begin{cases}E \varepsilon_{\max } & \text { for } \varepsilon_{\max }<\sigma_{y l}^{\prime} / E \\ \sigma_{y l}^{\prime} & \text { for } \varepsilon_{\max } \geq \sigma_{y l}^{\prime} / E\end{cases}
$$

i.e., by assuming ideal plasticity; $E$ is the Young's modulus, $\sigma_{y l}^{\prime}$ is the cyclic yield limit and $\varepsilon_{\max }$ is the maximum total strain. As seen in Fig. 1 a), the maximum stress is accurately described as ideal plastic at $450{ }^{\circ} \mathrm{C}$. The parameters $E$ and $\sigma_{y l}$ are given in Tab. 1

For $550^{\circ} \mathrm{C}$, on the other hand, the maximum stress is better described by a Ramberg-Osgood type equation

$$
\varepsilon_{\max }=\frac{\sigma_{\max }}{E}+\left(\frac{\sigma_{\max }}{K_{\max }^{\prime}}\right)^{1 / n_{\max }^{\prime}}
$$

with the material parameters $E, K_{\text {max }}^{\prime}$ and $n_{\text {max }}^{\prime}$ given in Tab. 1] Eqn. 7 is plotted in Fig. 1]a). From Fig. 1 a) it is evident that the material behaviour (with regard to maximum stress) changes between $450{ }^{\circ} \mathrm{C}$ and $550{ }^{\circ} \mathrm{C}$; low strains at $450{ }^{\circ} \mathrm{C}$ will produce a significant mean stress (due to high maximum stress) whereas, at $550{ }^{\circ} \mathrm{C}$, the mean stress is quite small at all strains. Some microstructural change may be the cause for this shift in behaviour; however, it is out of the scope of the current work to study this phenomenon. Here, it will
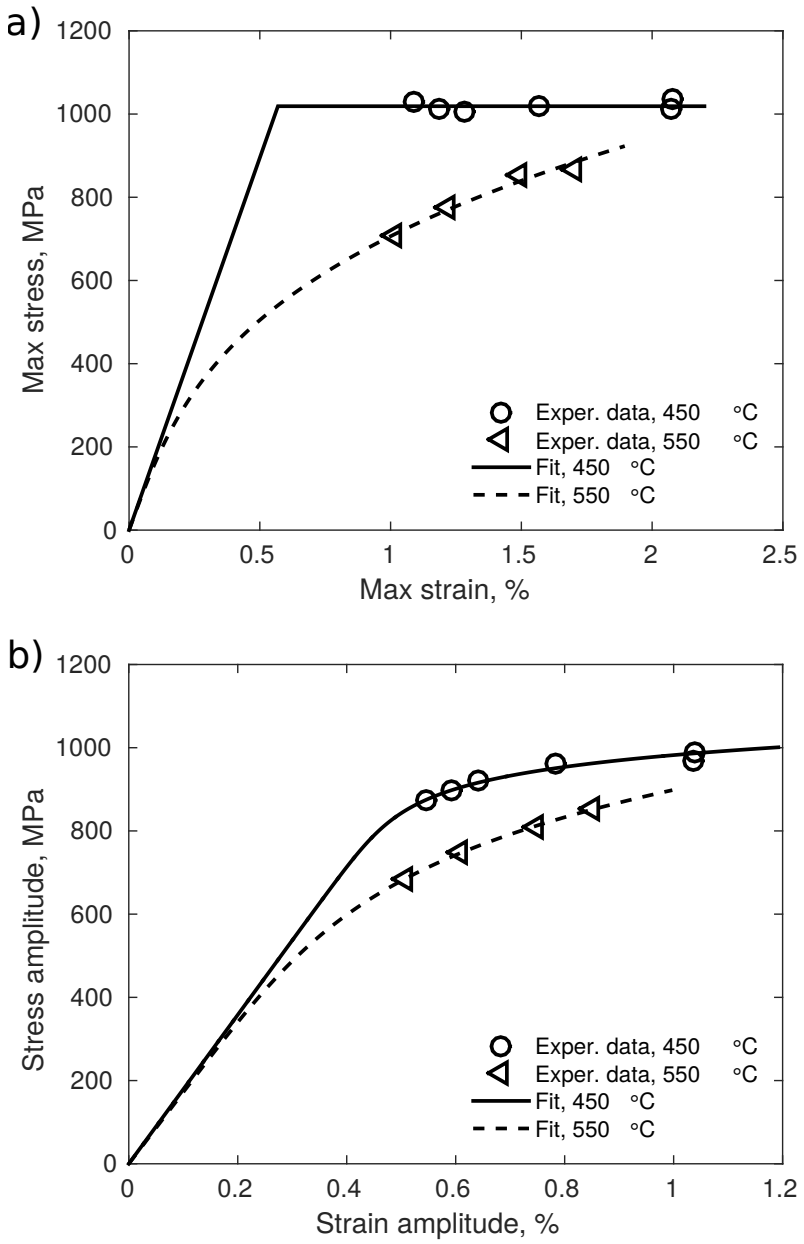

Figure 1. Material behaviour at $450{ }^{\circ} \mathrm{C}$ and $550{ }^{\circ} \mathrm{C}$ based on data from Ref. [7]. a) Maximum stress versus maximum strain. b) Stress amplitude versus strain amplitude.

simply be accepted that a shift in material behaviour exists between $450{ }^{\circ} \mathrm{C}$ and $550^{\circ} \mathrm{C}$ and that an accurate maximum stress can be predicted by assuming ideal plasticity at $450{ }^{\circ} \mathrm{C}$ but by using a Ramberg-Osgood type equation at $550^{\circ} \mathrm{C}$.

Secondly, the stress range, $\Delta \sigma$ is determined by assuming that the studied alloy follows Masing behaviour described by a Ramberg-Osgood type equation

$$
\Delta \varepsilon=\frac{\Delta \sigma}{E}+2\left(\frac{\Delta \sigma}{2 K^{\prime}}\right)^{1 / n^{\prime}}
$$

where $\Delta \varepsilon$ is the total strain range and the material parameters $E, K^{\prime}$ and $n^{\prime}$ are given in Tab. 1. Figure 1 b) shows Eqn. 8 fitted at both $450{ }^{\circ} \mathrm{C}$ and $550{ }^{\circ} \mathrm{C}$ (the figure is plotted in terms of amplitudes, i.e., $\Delta \sigma / 2$ and $\Delta \varepsilon / 2)$. The plastic strain range, $\Delta \varepsilon_{p}$, can then be determined as

$$
\Delta \varepsilon_{p}=\Delta \varepsilon-\frac{\Delta \sigma}{E}
$$

Figure 2 shows that the outlined method accurately predicts maximum and minimum stresses (and, consequently, the mean stress) for smooth specimens. 


\begin{tabular}{llllllll}
\hline$T,{ }^{\circ} \mathbf{C}$ & $E, \mathbf{G P a}$ & $\nu$ & $\sigma_{y l}^{\prime}, \mathbf{M P a}$ & $K_{\max }^{\prime}, \mathbf{M P a}$ & $n_{\max }^{\prime}$ & $K^{\prime}, \mathbf{M P a}$ & $n^{\prime}$ \\
\hline 450 & 178.97 & 0.32 & 1018.83 & - & - & 1328.84 & 0.0560 \\
\hline 550 & 172.25 & 0.33 & - & 3660.76 & 0.321 & 2406.08 & 0.184 \\
\hline
\end{tabular}

TABLE 1. Material parameters fitted to data from Ref. [7/9].
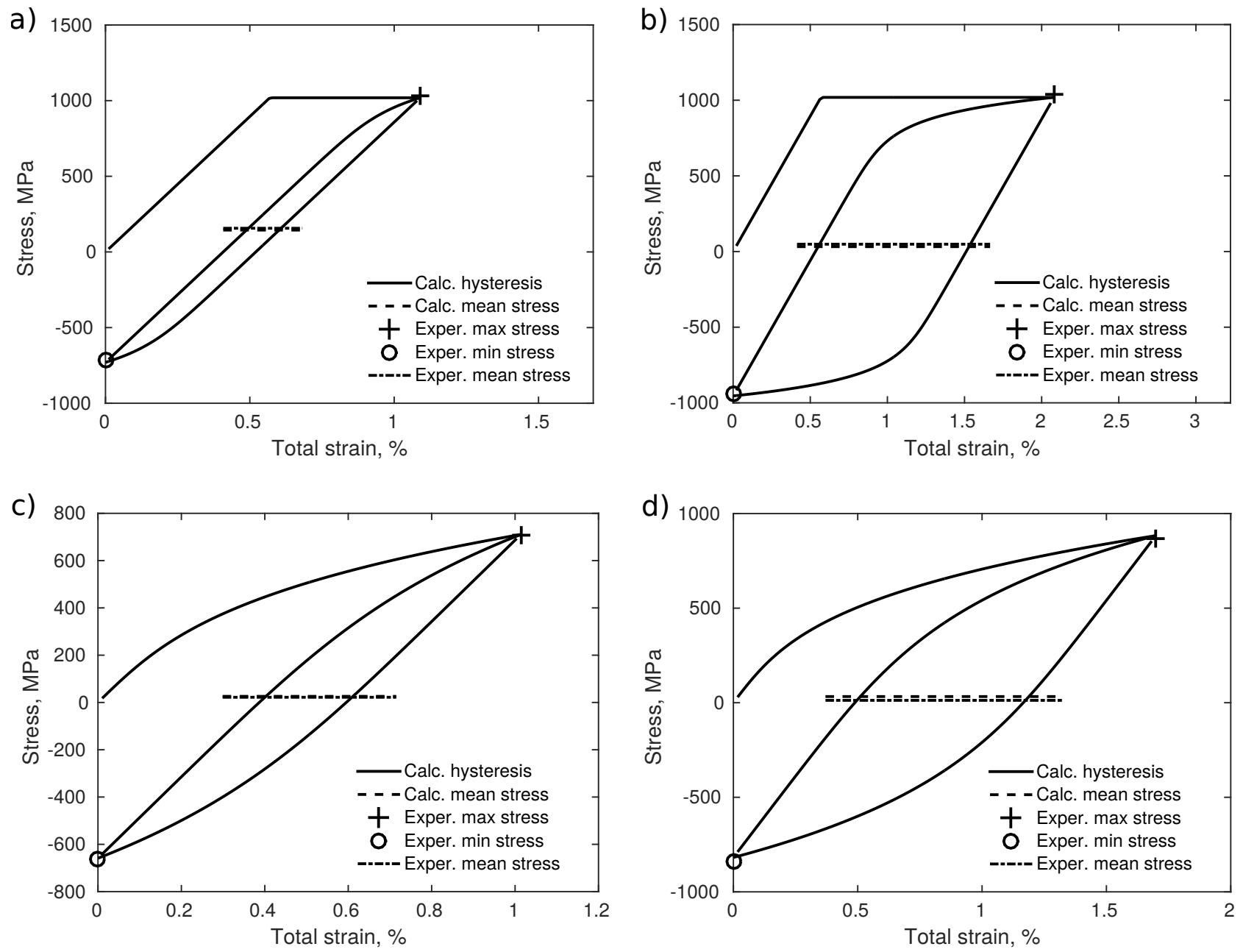

Figure 2. Comparison between experimentally determined stresses and calculated from Eqn. 6, 7 and 8 , for two different strain ranges at $450^{\circ} \mathrm{C}, \mathrm{a}$ ) and b), and at two different strain ranges at $550{ }^{\circ} \mathrm{C}, \mathrm{c}$ ) and d).

\subsection{FAtigue LifE MOdel FOR SMOOTH SPECIMENS}

Since mean stresses may not entirely relax (especially at $450^{\circ} \mathrm{C}$ for low load levels), the Smith-WatsonTopper (SWT) parameter [10], $\sigma_{\max } \Delta \varepsilon / 2$, is taken as the basis for the fatigue life model. The SWT parameter can be used for handling mean stress effects [10].

Here, since the majority of the studied specimens plasticise significantly, the variant of the SWT parameter suggested by Lorenzo and Laird [11] will be used. The assumption involved is that the fatigue life under mean stress can be correlated to the fully alternating case such that the parameter $\sigma_{\max } \Delta \varepsilon_{p} / 2$, for fatigue under mean stress, equals the parameter $\sigma_{a} \Delta \varepsilon_{p} / 2$, for the fully alternating load, where $\sigma_{a}$ is the stress amplitude. That is,

$$
\sigma_{\max } \frac{\Delta \varepsilon_{p}}{2}=\sigma_{a} \frac{\Delta \varepsilon_{p}}{2}
$$

which, with the Basquin equation,

$$
\sigma_{a}=\sigma_{f}^{\prime}\left(2 N_{f}\right)^{b}
$$

and the Manson-Coffin equation,

$$
\frac{\Delta \varepsilon_{p}}{2}=\varepsilon_{f}^{\prime}\left(2 N_{f}\right)^{c}
$$

yields

$$
\sigma_{\max } \frac{\Delta \varepsilon_{p}}{2}=\sigma_{f}^{\prime} \varepsilon_{f}^{\prime}\left(2 N_{f}\right)^{b+c}
$$

where $\sigma_{f}^{\prime}, \varepsilon_{f}^{\prime}, b$ and $c$ are fitting parameters which after merging yields

$$
\sigma_{\max } \frac{\Delta \varepsilon_{p}}{2}=A N_{f}^{a}
$$




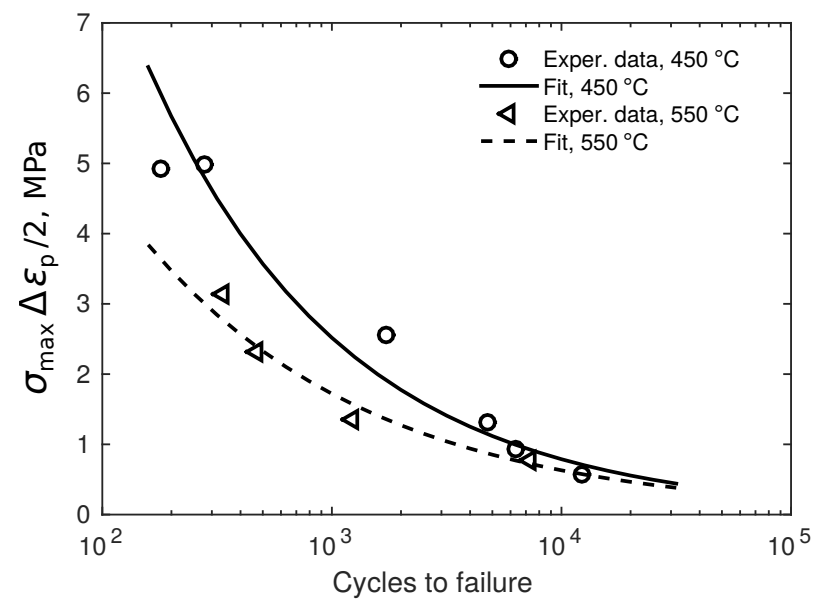

Figure 3. Fatigue life model calibration for smooth specimens at $450{ }^{\circ} \mathrm{C}$ and $550^{\circ} \mathrm{C}$. Fatigue life is described in terms of the modified SWT parameter, $\sigma_{\max } \Delta \varepsilon_{p} / 2$.

\begin{tabular}{lll}
\hline$T,{ }^{\circ} \mathbf{C}$ & $A, \mathbf{P a}$ & $a$ \\
\hline 450 & $81.94 \times 10^{6}$ & -0.504 \\
\hline 550 & $34.98 \times 10^{6}$ & -0.436 \\
\hline
\end{tabular}

TABLE 2. Fitted parameters in the fatigue life model for smooth specimens, see Eqn. 14

where $A$ and $a$ are fitting parameters. The parameter $\sigma_{\max } \Delta \varepsilon_{p} / 2$ will here be referred to as the modified SWT parameter.

The fatigue life model given by Eqn. 14 is calibrated with data from Moverare et al. [7] for smooth specimens. The modified SWT parameter is calculated from $\sigma_{\max }$ and $\Delta \varepsilon_{p} / 2$ determined as described above in the section Material model.

Figure 3 shows the fatigue life curves for smooth specimens at $450^{\circ} \mathrm{C}$ and $550{ }^{\circ} \mathrm{C}$ and the fitted parameters are given in Tab. 2

\section{EXPERIMENTAL WORK}

A test programme has been carried out involving LCF tests on blunt notched compact tension (CT) specimens made from the Ni base alloy 718 . The specimen geometry is shown in Fig. 4 and has outer dimensions of $32.4 \mathrm{~mm} \times 31.2 \mathrm{~mm} \times 12 \mathrm{~mm}$, where the latter is the thickness (i.e. it is the size of a halfinch CT specimen). Compared to a conventional CT specimen, the current specimen geometry differs in that it has a blunt notch (2 $\mathrm{mm}$ radius) to somewhat resemble a disk fir tree blade attachment.

The specimens were subjected to LCF load at $450{ }^{\circ} \mathrm{C}$ and $550^{\circ} \mathrm{C}$ for various load levels in the $4-10 \mathrm{kN}$ interval. The LCF tests were performed in load control with a load ratio of $R=0.05$. The tests were run until final rupture of the specimens.

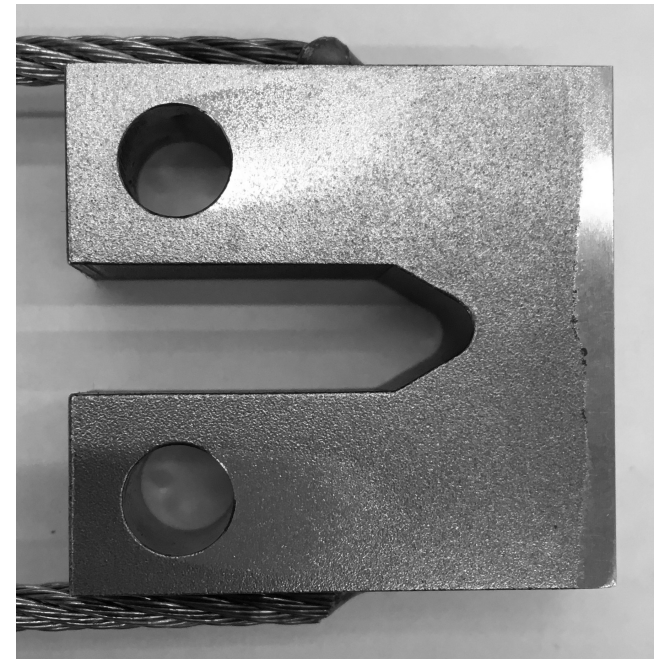

Figure 4. The blunt notch CT specimen used in the current study; image from Ref. 6].

\section{Results And Discussion}

\subsection{Stress AND STRAin RANGes FOR NOTCHED SPECIMENS}

The stress ahead of the notch is obtained from a linear elastic finite element analysis of the blunt notch CT specimen loaded with $1 \mathrm{~N}$ load. Stresses at all other load levels can be achieved by scaling the results from $1 \mathrm{~N}$ load. The stress' $y$-component is extracted as function of distance, $x$, from the notch as shown in Fig. 5. The $y$-component dominates the stress at the notch which enables the load to be treated as approximately uniaxial. Quantities obtained from the linear elastic finite element analysis will be denoted by an asterisk superscript; the stress ahead of the notch as function of $x$ is hence denoted as $\sigma^{*}(x)$, the stress range as $\Delta \sigma^{*}(x)$ and the notch root stress as $\sigma_{\text {root }}^{*}=\sigma^{*}(x=0)$. Figure 6 a) shows an example of the $\Delta \sigma^{*}(x)$ profile for $9.9 \mathrm{kN}$ applied load at $450^{\circ} \mathrm{C}$.

A Glinka shakedown is performed on the $\sigma^{*}(x)$ profile to get $\sigma_{\max }$ and $\Delta \varepsilon_{p} / 2$. A Glinka shakedown has previously been shown to give accurate results for this material and specimen geometry, see Ref. [6]. To get the maximum stress, the following equation systems are solved. For $450^{\circ} \mathrm{C}, \sigma_{\max }$ is the outcome of solving

$$
\left\{\begin{array}{l}
\int_{0}^{\varepsilon_{\max }} \sigma d \varepsilon=\frac{K_{f}^{2}\left(\sigma^{*}\right)^{2}}{2 E} \\
\sigma= \begin{cases}E \varepsilon & \text { for } \varepsilon<\sigma_{y l} / E \\
\sigma_{y l} & \text { for } \varepsilon \geq \sigma_{y l} / E\end{cases}
\end{array}\right.
$$

for $\sigma$ and for $550^{\circ} \mathrm{C}, \sigma_{\max }$ is the outcome of solving

$$
\left\{\begin{array}{l}
\int_{0}^{\varepsilon_{\max }} \sigma d \varepsilon=\frac{K_{f}^{2}\left(\sigma^{*}\right)^{2}}{2 E} \\
\varepsilon=\frac{\sigma}{E}+\left(\frac{\sigma}{K_{\max }^{\prime}}\right)^{1 / n_{\max }^{\prime}}
\end{array}\right.
$$

for $\sigma$. The stress range and the total strain range are 


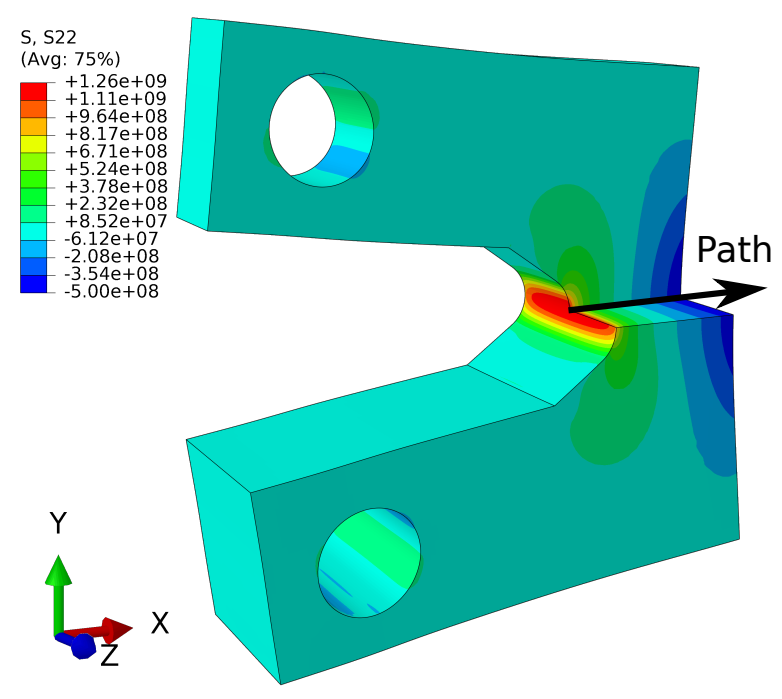

Figure 5. Path at which the $y$-component stress profile is extracted from a linear elastic finite element analysis; image from Ref. [6].

obtained by solving

$$
\left\{\begin{array}{l}
\int_{0}^{\Delta \varepsilon} \sigma d \varepsilon=\frac{K_{f}^{2}\left(\Delta \sigma^{*}\right)^{2}}{2 E} \\
\Delta \varepsilon=\frac{\Delta \sigma}{E}+2\left(\frac{\Delta \sigma}{2 K^{\prime}}\right)^{1 / n^{\prime}}
\end{array}\right.
$$

which enables the plastic strain range to be calculated from Eqn.9. Since $\sigma^{*}(x)$ is the local stress, the fatigue notch factor, $K_{f}$, is set to $K_{f}=1$ in Eqn. 15 16 and 17. Figure 6 shows an example of the thus achieved stress and strain ranges.

\subsection{Comparison of the FAtigue LifE OF SMOOTH AND NOTCHED SPECIMENS}

Figure 7 shows the modified SWT parameter plotted versus the fatigue life for both smooth and blunt notch CT specimens; for the latter, the modified SWT parameter has been calculated at the notch root. It is evident that, at both $450^{\circ} \mathrm{C}$ and $550{ }^{\circ} \mathrm{C}$, the predicted life of the notched specimens would be too conservative if based on fatigue life curves for smooth specimens and the notch root modified SWT parameter. That is, notch support needs to be included.

As seen in Fig. 7, the notch support diminishes for low values of the modified SWT parameter (roughly at $10^{4}$ cycles to rupture). The low values of the modified SWT parameter are caused by the plastic strain range approaching zero for low loads. The diminishing of the notch support could therefore be said to occur roughly at the transition into the high cycle fatigue region.

To accurately account for notches in life prediction in the low cycle regime, the theory of critical distances will be applied. In the following sections, the distance from the notch at which the modified SWT parameter coincides with that of a smooth specimen of the same fatigue life will be identified and used to calibrate an equation for establishing the critical distance.
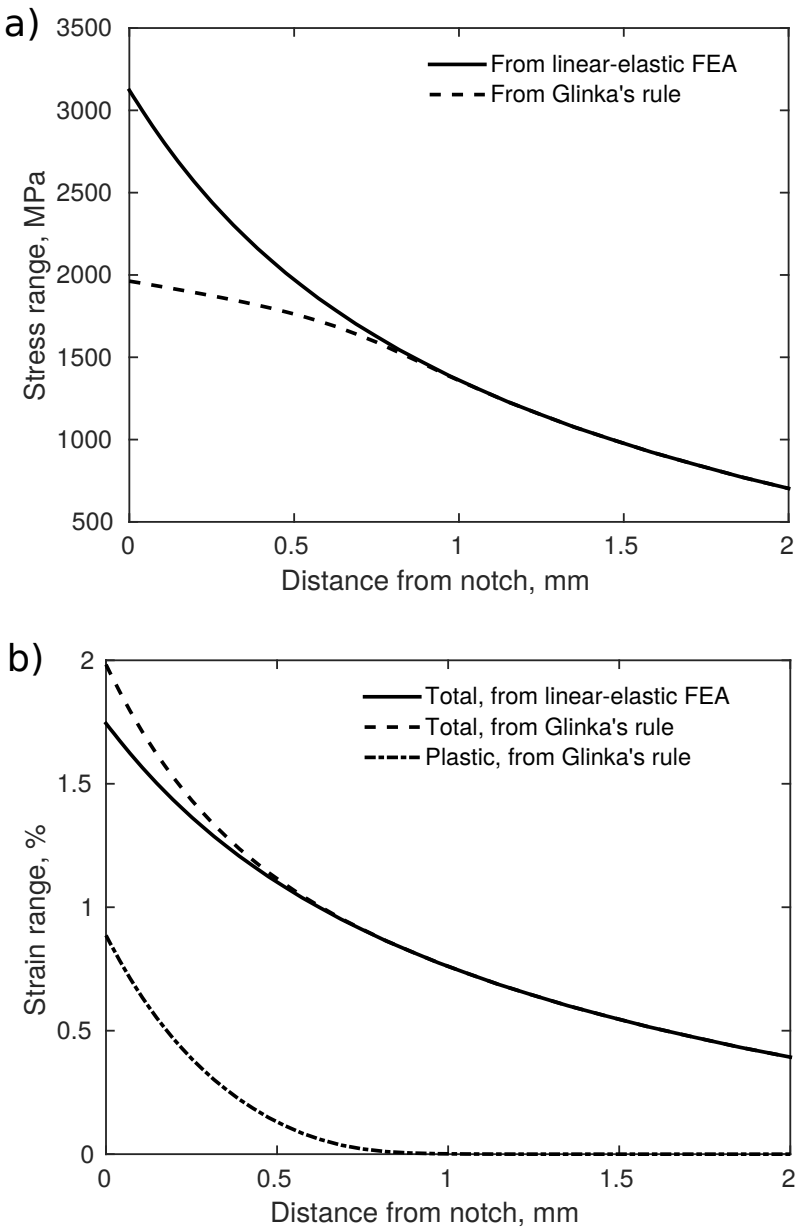

Figure 6. Stress and strain ranges from a linearelastic finite element analysis (FEA) at $450{ }^{\circ} \mathrm{C}$ and $9.9 \mathrm{kN}$ load subjected to a Glinka shakedown. a) Stress range as function of the distance from the notch. b) Total and plastic strain ranges as function of the distance from the notch. Figure from Ref. [6].

\subsection{A pragmatic EQUation for the CRITICAL DISTANCE}

Since Eqn. 4 has gained some use for the critical distance approach in fatigue (see the Introduction section), it will be taken as basis also in the current work. Although useful, Eqn. 4 is somewhat inconvenient as it requires an iterative process to solve (since $N_{f}$ may not be known a priori). Therefore, Eqn. 4 will be adopted on a form which enables the critical distance to be calculated directly from notch root variables (from an elastic FEA). A rationale for this is provided here.

Firstly, $N_{f}$ can be eliminated in Eqn. 4 by using Eqn. 14 giving

$$
\sigma_{\max }(r) \frac{\Delta \varepsilon_{p}(r)}{2}=A\left(C_{1} r^{c_{1}}\right)^{a}=C_{2} r^{c_{2}}
$$

In order to relate plastic strain with stress from an elastic finite element analysis, Glinka's rule is used

$$
\int_{0}^{\Delta \varepsilon_{p}(r) / 2} \sigma(r) d \varepsilon=\frac{K_{f}^{2}\left(\sigma^{*}(r)\right)^{2}}{2 E}
$$



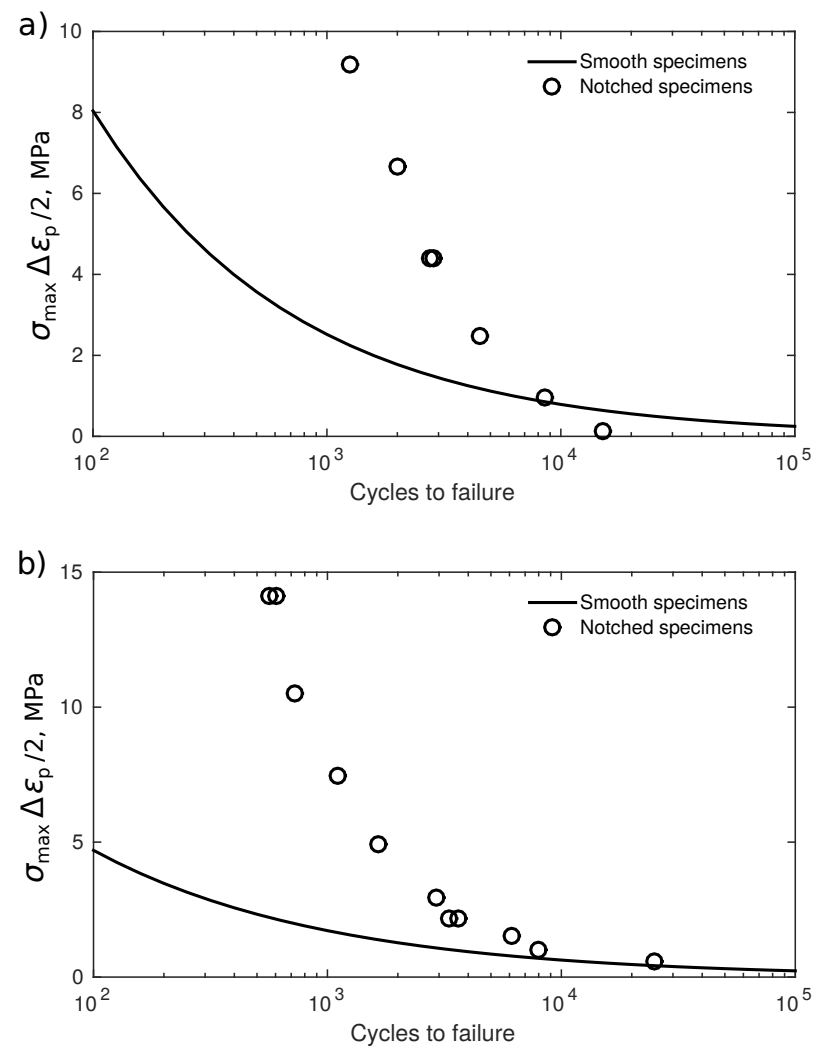

Figure 7. Comparison between the fatigue lives of smooth and notched specimens (data for smooth specimens are given as a fitted curve and data for notched specimens are experimental data). The fatigue life is described in terms of the modified SWT parameter, $\sigma_{\max } \Delta \varepsilon_{p} / 2$, which, for the notched specimens, has been calculated at the notch root: a) $450{ }^{\circ} \mathrm{C}$ and b) $550^{\circ} \mathrm{C}$.

where $\sigma^{*}(r)$ is the stress taken from an elastic finite element analysis the distance $r$ from the notch root. Since $\sigma^{*}$ represent the local stress, the fatigue notch factor is set to $K_{f}=1$.

For simplicity, rigid-ideal plastic material behaviour is assumed (i.e. $\sigma=\sigma_{y l}$ and $\varepsilon=\varepsilon_{p}$ ) which in Eqn. 19 gives

$$
\sigma_{y l} \frac{\Delta \varepsilon_{p}(r)}{2}=\frac{\left(\sigma^{*}(r)\right)^{2}}{2 E}
$$

and, in Eqn. 18

$$
\sigma_{y l} \frac{\Delta \varepsilon_{p}(r)}{2}=C_{2} r^{c_{2}}
$$

Equating Eqn. 20 and 21 yields

$$
C_{2} r^{c_{2}}=\frac{\left(\sigma^{*}(r)\right)^{2}}{2 E} \Rightarrow r=\left[\sqrt{\frac{1}{2 E C_{2}}} \sigma^{*}(r)\right]^{2 / c_{2}}
$$

or, after merging constant parameters,

$$
r=C_{3}\left(\sigma^{*}(r)\right)^{c_{3}}
$$

Assuming that the maximum stress occurs at the notch root and the critical distance of interest is close enough to the notch root (which it typically is), a sufficient approximation of the stress at the distance $r$ ahead of the notch would be

$$
\sigma^{*}(r)=\sigma_{\text {root }}^{*}(1+\chi r)
$$

which is a linear approximation of the stress in terms of the relative stress gradient, $\chi$, taken as

$$
\chi=\left.\frac{1}{\sigma_{\text {root }}^{*}} \frac{\partial \sigma^{*}}{\partial x}\right|_{x=0}
$$

i.e. the stress gradient evaluated at the notch root normalised with the notch root stress. The approximation of the stress ahead of the notch can be improved by averaging $\chi$ over a distance roughly equal to the expected critical distance. Therefore, $\chi$ will be replaced by an average relative stress gradient, $\bar{\chi}$, according to

$$
\bar{\chi}=\left.\frac{1}{\sigma_{\text {root }}^{*}} \frac{\Delta \sigma^{*}}{\Delta x}\right|_{x=0}
$$

where $\Delta x$ is taken as roughly the same as the expected critical distance. Based on previous experiences [6], the critical distance should be in the order of a few tenths of a millimetre and is here (arbitrarily) set to $\Delta x \approx 0.4 \mathrm{~mm}$.

Inserting Eqn. 24 in Eqn. 23 (with $\bar{\chi}$ substituted for $\chi$ ) gives

$$
r=C_{3}\left(\sigma_{\text {root }}^{*}(1+\bar{\chi} r)\right)^{c_{3}}
$$

or, alternatively,

$$
\sigma_{\text {root }}^{*}=\frac{D r^{d}}{1+\bar{\chi} r}
$$

with $D=\left(1 / C_{3}\right)^{1 / c_{3}}$ and $d=1 / c_{3}$.

Equation 28 is used in the current work to correlate the critical distance with the notch root stress from a linear elastic FEA. Although several simplifications and approximations were introduced in the derivation of Eqn. 28, it is believed to be, at least, a good starting point for finding such a relation. Equation 28 is sigmoid shaped and have some characteristics which seem intuitively reasonable: 1) For small values of $\sigma_{\text {root }}^{*}, r$ is approximately zero (in agreement with the notch support diminishing for long lives, see Fig. 7). 2) The critical distance, $r$, asymptotically approaches $1 /(-\bar{\chi})$ for large values of $\sigma_{\text {root }}^{*}$; it does seem reasonable that an upper limit in $r$ should exist and be determined by the notch stress gradient.

It should be noted that, due to the involved assumptions of material behaviour, the parameters $D$ and $d$ are material and temperature dependent.

\subsection{Calibration of the CRitical distance}

A few data points from $450{ }^{\circ} \mathrm{C}$ and $550{ }^{\circ} \mathrm{C}$ were used for calibrating the critical distance equation (Eqn. 28). By examining Eqn. 28, one realises that the upper limit of the critical distance is determined by the factor $1 /(1+\bar{\chi} r)$ which causes $r$ to asymptotically approach $r=1 /(-\bar{\chi})$ for large $\sigma_{\text {root }}^{*}$. Therefore, the calibration 


\begin{tabular}{lll}
\hline$T,{ }^{\circ} \mathbf{C}$ & $D, \mathbf{P a} / \mathbf{m}^{d}$ & $d$ \\
\hline 450 & $3.3045 \times 10^{9}$ & 0.0493 \\
\hline 550 & $4.9181 \times 10^{9}$ & 0.1396 \\
\hline
\end{tabular}

TABlE 3. Fitted parameters for Eqn. 28. The fitting was done with $\sigma_{\text {root }}^{*}$ in $\mathrm{Pa}$ and $r$ in $\mathrm{m}$.

should be possible with just a few data points taken at low values of $\sigma_{\text {root }}^{*}$ (since the remaining $r-\sigma_{\text {root }}^{*}$ curve will be dominated by the $1 /(1+\bar{\chi} r)$ factor $)$.

The calibration is done as follows:

(1.) For each calibration point, the experimentally observed fatigue life of the notched specimen, $N_{f}^{\text {notch }}$, is given.

(2.) A target modified SWT parameter, $\hat{\sigma}_{\max } \Delta \hat{\varepsilon}_{p} / 2$, is determined from the $\sigma_{\max } \Delta \varepsilon_{p} / 2-N_{f}$ curve for smooth specimens (i.e. from Eqn. 14) as

$$
\hat{\sigma}_{\max } \Delta \hat{\varepsilon}_{p} / 2=A\left(N_{f}^{\text {notch }}\right)^{a}
$$

(3.) The critical distance, $r$, is determined so that

$$
\sigma_{\max }(x=r) \Delta \varepsilon_{p}(x=r) / 2=\hat{\sigma}_{\max } \Delta \hat{\varepsilon}_{p} / 2
$$

which gives $r ; \sigma_{\max }(x)$ and $\Delta \varepsilon_{p}(x)$ profiles are obtained by Glinka shakedown as shown in Fig. 6

(4.) The hence found $r$ values are plotted versus the corresponding $\sigma_{\text {root }}^{*}$ value and the parameters $D$ and $d$ in Eqn. 28 are fitted.

Figure 8 shows the critical distance (obtained as described above) versus the notch root stress, $\sigma_{\text {root }}^{*}$. At both $450{ }^{\circ} \mathrm{C}$ and b) $550^{\circ} \mathrm{C}$, the three points with lowest $\sigma_{\text {root }}^{*}$ were used to fit Eqn. 28 As seen, Eqn. 28 (when extrapolated) describes the critical distances for the remaining data points with reasonable accuracy. At $550{ }^{\circ} \mathrm{C}$, there is a slight discrepancy between Eqn. 28 and experimental data for high $\sigma_{\text {root }}^{*}$ values; most likely due to the critical distance exceeding the distance over which $\bar{\chi}$ was average, causing the approximation of the stress ahead of the notch to break down. However, the discrepancy is relatively minor.

Overall, Eqn. 28 captures the critical distance with reasonable accuracy and will be used for predicting the fatigue life of notched specimens. The fitting parameters in Eqn. 28 are given in Tab. 3 .

\subsection{Prediction of the FAtigue Life of NOTCHED SPECIMENS}

The calibrated equation for the critical distance (Eqn. 28) can now be used together with the fatigue life model for smooth specimens (Eqn. 14) to make a prediction of the fatigue life of notched specimens. It should be noted, though, that the notch support only is present in the LCF regime.

The prediction is done as outlined below: a)

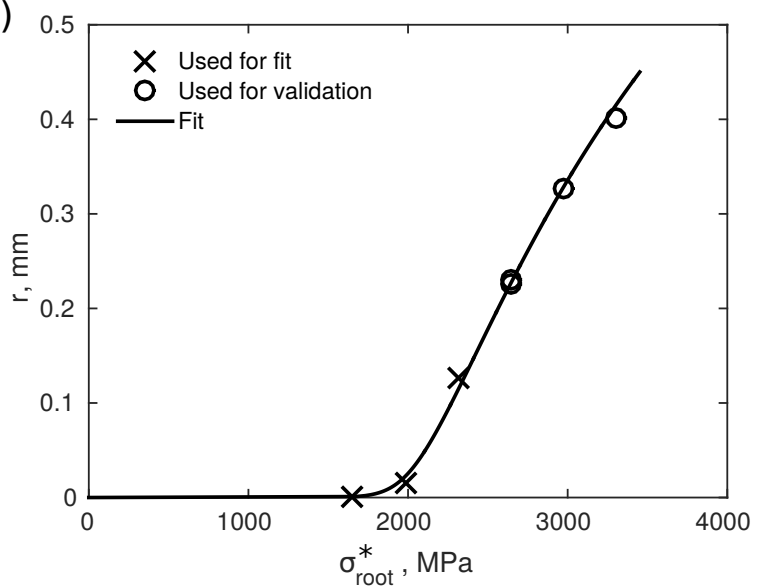

b)

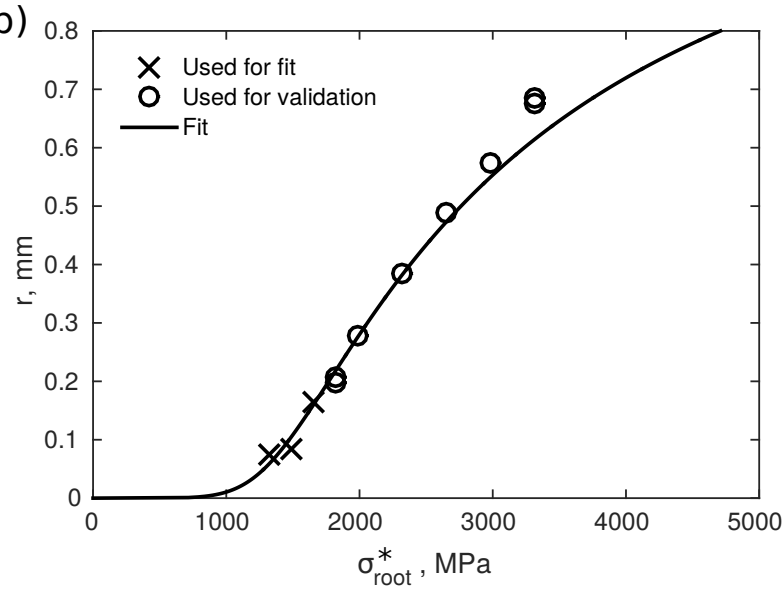

Figure 8. The critical distance, $r$, as function of the notch root stress from an elastic finite element analysis, $\sigma_{\text {root }}^{*}$, for: a) $450^{\circ} \mathrm{C}$ and b) $550{ }^{\circ} \mathrm{C}$. The fit was done using Eqn. 28 a few points taken at low $\sigma_{\text {root }}^{*}$ were used for calibration.

(1.) For each notched specimen, the $\sigma^{*}(x)$ profile is determined from a linear elastic finite element analysis. The parameters $\sigma_{\text {root }}^{*}$ and $\bar{\chi}$ are established from the $\sigma^{*}(x)$ profile.

(2.) The critical distance, $r$, is determined from Eqn. 28 with values from Tab. 3

(3.) The stress $\sigma^{*}(r)$ is retrieved from the $\sigma^{*}(x)$ profile and a Glinka shakedown is performed (see Eqn. 15 16 and 17), giving $\sigma_{\max }$ and $\Delta \varepsilon_{p}$. This allows the modified SWT parameter, $\sigma_{\max } \Delta \varepsilon_{p} / 2$, to be calculated at $r$.

(4.) The hence obtained modified SWT parameter is used in conjunction with fatigue life data for smooth specimens (see Eqn. 14) to predict the life of notched specimens.

Figure 9 shows the results of the fatigue life prediction of notched specimens as outlines above. As in Fig. 7, data from the notched specimens has been plotted in terms of the notch root modified SWT parameter, but the prediction has been made based on $\sigma_{\max }(x=r) \Delta \varepsilon_{p}(x=r) / 2$.

The fatigue life prediction based on the critical 

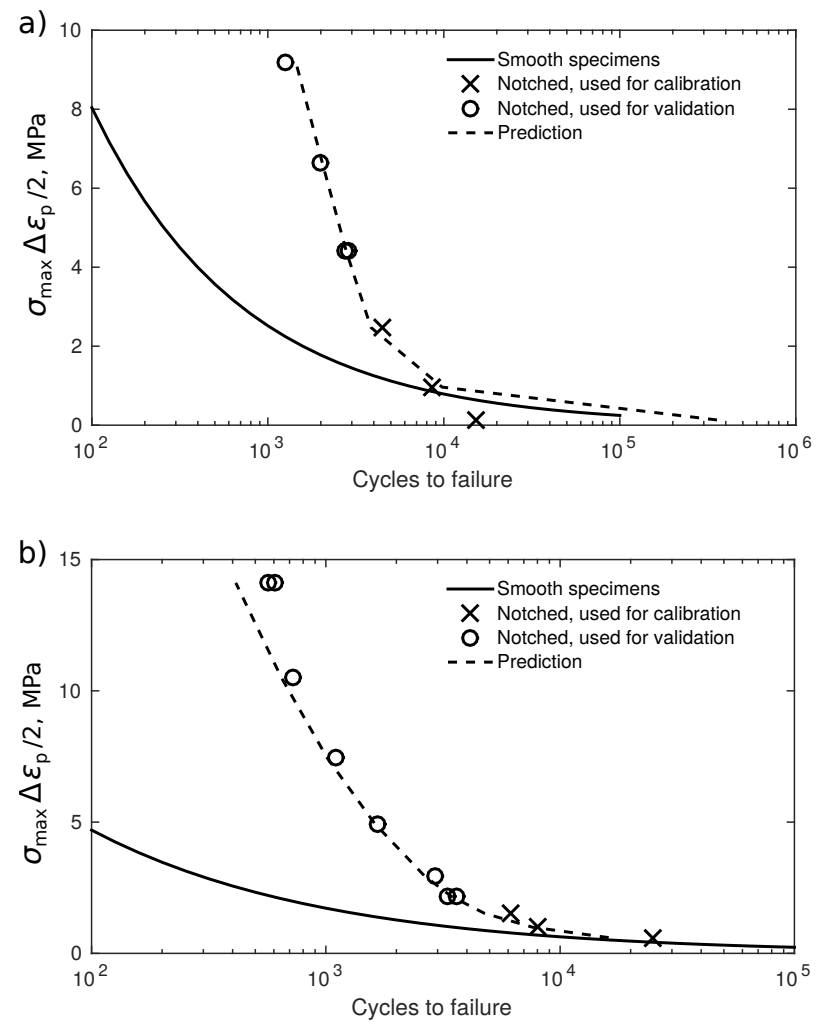

Figure 9. The modified SWT parameter, $\sigma_{\max } \Delta \varepsilon_{p} / 2$, versus cycles to failure for smooth and notched specimens at: a) $450{ }^{\circ} \mathrm{C}$ and b) $550^{\circ} \mathrm{C}$. For the notched specimens, the data is plotted versus the notch root modified SWT parameter, $\sigma_{\max }(x=0) \Delta \varepsilon_{p}(x=0) / 2$, but the prediction has been made based on the modified SWT parameter at the critical distance, $r$, i.e., $\sigma_{\max }(x=r) \Delta \varepsilon_{p}(x=r) / 2$.

distance obtained from Eqn. 28 agrees well with experimental results (see Fig. 9 thus indicating that the suggested equation for the critical distance (Eqn. 28) certainly is usable. In addition, it should be noted that only a limited number of data points were needed to calibrate Eqn.28, making it a fairly efficient approach to account for notch support.

It should be noted, however, that the applicability of the life model is limited to loads which gives, at least some, plastic strain. The specimen with the longest life in Fig. 9 agrees less well with the prediction due to the modified SWT parameter being essentially zero.

\section{Conclusions}

Low cycle fatigue tests were performed on blunt notch compact tension specimens made from alloy 718 . The results indicated that notch support needed to be incorporated in the lifing method not to cause an overly conservative life prediction. The notch support, however, diminished as the plastic strain range decreased, indicating that notch support is only present in the low cycle fatigue regime for this alloy.

To account for the notch support, a critical distance approach was attempted. An equation, relating the critical distance to the notch root stress from a lin- ear elastic finite element analysis, was derived. The chosen life model was formulated in terms of a variation on the Smith-Watson-Topper parameter, the modified SWT parameter (based on the plastic strain range). The modified SWT parameter taken at the critical distance was used in a life model calibrated for smooth specimens to predict the fatigue life of notched specimens. The fatigue life prediction for notched specimens agreed well with available experimental data.

In summary, the current work has shown that:

(1.) A critical distance approach can be applied to account for notch support in alloy 718 .

(2.) The critical distance can be correlated to the notch root stress from a linear elastic finite element analysis, considerably simplifying its use.

(3.) The suggested equation for the critical distance requires relatively few data points for calibration.

\section{LIST OF SYMBOLS}

$A$ Fatigue life model coefficient $[\mathrm{Pa}]$

$D$ Coefficient in equation for the critical distance $\left[\mathrm{Pa} / \mathrm{m}^{d}\right]$

$E \quad$ Young's modulus $[\mathrm{Pa}]$

$K^{\prime}$ Cyclic Ramberg-Osgood coefficient $[\mathrm{Pa}]$

$K_{\max }^{\prime}$ Cyclic Ramberg-Osgood coefficient for determining max stress/strain $[\mathrm{Pa}]$

$K_{c}$ Fracture toughness $[\mathrm{Pa} \sqrt{\mathrm{m}}]$

$K_{t h}$ Threshold stress intensity factor for crack growth $[\mathrm{Pa} \sqrt{\mathrm{m}}]$

$K_{f}$ Fatigue notch factor

$N_{f}$ Cycles to failure

$N_{f}^{\text {notch }}$ Experimentally observed cycles to failure for notched specimens

$R$ Load ratio, minimum load over maximum load

$R_{\varepsilon}$ Strain ratio, minimum strain over maximum strain

$T$ Temperature $\left[{ }^{\circ} \mathrm{C}\right]$

a Fatigue life model exponent

$b \quad$ Fatigue strength exponent

$c$ Fatigue ductility exponent

$d$ Exponent in equation for the critical distance

$n^{\prime}$ Cyclic Ramberg-Osgood exponent

$n_{\max }^{\prime}$ Cyclic Ramberg-Osgood exponent for determining max stress/strain

$r$ Critical distance $[\mathrm{m}]$

$\Delta \varepsilon$ Total strain range

$\Delta \varepsilon_{p} \quad$ Plastic strain range

$\Delta \varepsilon_{p}^{\text {root }}$ Notch root plastic strain range

$\Delta \sigma$ Stress range $[\mathrm{Pa}]$

$\Delta \sigma^{*}$ Stress range from a linear elastic analysis [Pa]

$\varepsilon_{p} \quad$ Plastic strain

$\varepsilon_{\max }$ Maximum total strain

$\nu$ Poisson's ratio

$\sigma_{0}$ Critical value of stress $[\mathrm{Pa}]$

$\sigma^{*}$ Stress from a linear elastic analysis $[\mathrm{Pa}]$

$\sigma_{\text {root }}^{*}$ Notch root stress from a linear elastic analysis $[\mathrm{Pa}]$ 
$\sigma_{y l} \quad$ Yield limit $[\mathrm{Pa}]$

$\sigma_{y l}^{\prime} \quad$ Cyclic yield limit $[\mathrm{Pa}]$

$\sigma_{\max }$ Maximum stress $[\mathrm{Pa}]$

$\sigma_{a}$ Stress amplitude $[\mathrm{Pa}]$

$\sigma_{f}^{\prime} \quad$ Fatigue strength coefficient $[\mathrm{Pa}]$

$\sigma_{\max } \Delta \varepsilon_{p} / 2$ Modified SWT parameter [Pa]

$\hat{\sigma}_{\text {max }} \Delta \hat{\varepsilon}_{p} / 2$ Target modified SWT parameter $[\mathrm{Pa}]$

$\chi \quad$ Relative stress gradient $[1 / \mathrm{m}]$

$\bar{\chi} \quad$ Averaged relative stress gradient $[1 / \mathrm{m}]$

\section{ACKNOWLEDGEMENTS}

This project has received funding from the European Union's Horizon 2020 research and innovation programme under grant agreement No. 653941.

\section{REFERENCES}

[1] R. C. Reed. The Superalloys: Fundamentals and Applications. Cambridge University Press, 2006. DOI:10.1017/CBO9780511541285.

[2] L. Mäde, H. Gottschalk, S. Schmitz, et al. Probabilistic LCF Risk Evaluation of a Turbine Vane by Combined Size Effect and Notch Support Modeling. In ASME Turbo Expo 2017: Turbomachinery Technical Conference and Exposition, vol. 7A: Structures and Dynamics. 2017. DOI:10.1115/gt2017-64408.

[3] C. Kontermann, H. Almstedt, A. Scholz, M. Oechsner. Notch Support for LCF-Loading: A Fracture Mechanics Approach. Procedia Structural Integrity 2:3125 - 3134, 2016. 21st European Conference on Fracture, ECF21, 20-24 June 2016, Catania, Italy, DOI:10.1016/j.prostr.2016.06.390.

[4] D. Taylor (ed.). The Theory of Critical Distances. Elsevier Science Ltd, Oxford, 2007. DOI:10.1016/b978-0-08-044478-9.x5000-5
[5] D. Leidermark, J. Moverare, K. Simonsson, S. Sjöström. A combined critical plane and critical distance approach for predicting fatigue crack initiation in notched single-crystal superalloy components. International Journal of Fatigue 33(10):1351 - 1359, 2011. DOI:10.1016/j.ijfatigue.2011.05.009

[6] R. Eriksson, K. Simonsson, D. Leidermark, J. Moverare. Evaluation of notch effects in low cycle fatigue of alloy 718 using critical distances. MATEC Web of Conferences 165:15001, 2018. DOI:10.1051/matecconf/201816515001

[7] J. Moverare, G. Leijon, H. Brodin, F. Palmert. Effect of SO2 and water vapour on the low-cycle fatigue properties of nickel-base superalloys at elevated temperature. Materials Science and Engineering A 564:107-115, 2013. DOI:10.1016/j.msea.2012.11.079.

[8] M. Fukuhara, A. Sanpei. Elastic moduli and internal frictions of Inconel 718 and Ti-6Al-4V as a function of temperature. Journal of Materials Science Letters 12(14):1122-1124, 1993. DOI:10.1007/BF00420541

[9] H. Ledbetter. Temperature behaviour of Young's moduli of forty engineering alloys. Cryogenics 22(12):653 - 656, 1982. DOI:10.1016/0011-2275(82)90072-8

[10] K. Smith, T. Topper, P. Watson. A stress-strain function for the fatigue of metals (stress-strain function for metal fatigue including mean stress effect). $J$ Materials 5:767-778, 1970.

[11] F. Lorenzo, C. Laird. A new approach to predicting fatigue life behavior under the action of mean stresses. Materials Science and Engineering 62(2):205 - 210, 1984. DOI:10.1016/0025-5416(84)90223-4 